\title{
Swiss Medical Board - cui bono?
}

\author{
Thomas Cerny ${ }^{a}$, \\ Jakob Passweg , \\ Giorgio Nosedac

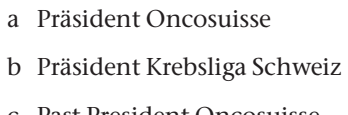 \\ c Past President Oncosuisse
}

Interessenbindungen: keine

Eine Stellungnahme des Swiss Medical Board findet sich im Anschluss an diesen Beitrag auf S. 1729.
Korrespondenz:

Prof. Dr. med. Thomas Cerny Oncosuisse

Effingerstrasse 40

CH-3008 Bern

Tel. 0313899100

Fax 0313899160

thomas.cerny[at]kssg.ch
Mit einer gewissen Regelmässigkeit lösen die Berichte des Swiss Medical Boards (SMB) heftige Reaktionen aus. Dies erstaunt an sich nicht, greift doch das SMB Themen mit Konfliktpotential auf. So etwa im Februar 2014 mit einem Bericht über Mammographie-Programme [1] oder diesen April im Zusammenhang mit der Primärprävention kardiovaskulärer Erkrankungen mit Statinen [2]. Es würde jedoch zu kurz greifen, die teilweise heftigen Reaktionen auf reine Verteidigung von Interessen $\mathrm{zu}$ reduzieren. Vielmehr stehen auch die Zusammensetzung, die Arbeitsweise und das Vorgehen - sprich die Professionalität - des SMB wie auch die Themenauswahl respektive deren Abgrenzung in der Kritik.

\section{Gleiche Daten - andere Schlussfolgerungen}

Im Zusammenhang mit dem Bericht zum systematischen Mammographie-Screening im Rahmen von Programmen empfahl das SMB, keine neuen Mammographie-Screening-Programme einzuführen und die bestehenden zu befristen. Darüber hinaus seien alle Formen des Mammographie-Screenings zu evaluieren. Zudem riet das SMB zu einer vorgängigen, gründlichen ärztlichen Abklärung und einer verständlichen Aufklärung der Frauen.

Auch dieser Bericht des SMB wurde heftig kritisiert. Er enthält keine neuen Daten, die Studien und deren Resultate waren seit vielen Jahren bekannt. Die vom SMB durchgeführte Kosten-Nutzen-Analyse entspricht in keiner Art und Weise den Anforderungen an Evaluationen von medizinischen Interventionen allgemein. Interessanterweise wurden die Resultate der Kosten-Nutzen-Rechnung auch vom SMB nicht als Argument ins Feld geführt.

Zusammengefasst sind wir der Ansicht, dass das SMB die positiven Auswirkungen des Mammographie-Screenings unterschätzt und die unerwünschten Auswirkungen überschätzt (siehe auch Kasten, Beurteilung von H. de Koning). Auch die englische Expertengruppe, die 2012 eine viel beachtete, differenzierte Review [3] zum Nutzen des seit 1988 bestehenden Mammographie-Screenings publiziert hat, ist zum Schluss gekommen, dass die Programme einen relevanten Nutzen bringen und fortgeführt werden sollen. Interessanterweise gibt das SMB die englische Publikation als wichtige Grundlage ihres eigenen Berichts an - dennoch kommt es zu gegensätzlichen Schlussfolgerungen als das englische Expertengremium.

Eine Woche vor der Publikation des SMBBerichts veröffentlichte das «Health Council of the Netherlands» im Januar 2014 einen umfangreichen

\section{Zusammenfassung}

Im Februar 2014 hat das Swiss Medical Board (SMB) einen Bericht zum Mammographie-Screening veröffentlicht. Dabei kam das SMB zum Schluss, dass der Schaden des Screenings grösser ist als der Nutzen. Der SMB-Bericht wurde heftig kritisiert: Die Analyse weist gravierende Mängel auf. Insgesamt werden die positiven Auswirkungen unterschätzt, die negativen Folgen überschätzt. Das Subsummieren von falschpositiven Resultaten und Überdiagnosen - zwei bekanntermassen vollkommen unterschiedliche Situationen - unter dem Begriff «Fehlbefunde» zeigt beispielhaft die Unzulänglichkeit. Ein Einbezug der befragten Experten hätte solche Fehler verhindert.

Das SMB hat sich in einer Post-Publication-Review zur Kritik geäussert. Es beurteilt die Sachlage als «unverändert» und bleibt bei seinen Empfehlungen. Wir halten darum erneut fest: Das SMB hat einseitig das Mammographie-Screening in Programmen analysiert. Neue Daten und die Realität der opportunistischen Früherkennung blieben unberücksichtigt, die Meinung befragter Experten ebenfalls. Stattdessen wurden eine nicht nachvollziehbare Kosten-Nutzen-Analyse durchgeführt und widersprüchliche Empfehlungen formuliert.

Auch wir sind der Meinung, dass das Mammographie-Screening kontinuierlich gemäss aktuellen wissenschaftlichen Erkenntnissen weiterentwickelt werden soll. Dazu braucht es qualitativ gute und differenzierte Grundlagen. Der Bericht des SMB zum Mammographie-Screening erfüllt diese Anforderung nicht.

Bericht über das niederländische MammographieProgramm [4]. Auch diese Expertengruppe sprach sich für die Fortführung der Programme aus.

Die Empfehlungen des SMB sind widersprüchlich. Entscheidet sich nämlich eine Frau nach gründlicher ärztlicher Abklärung und einer verständlichen Aufklärung dafür, eine Früherkennungs- 
Mammographie durchzuführen, müsste diese nach der Logik des SMB - d.h. nach Beendigung der Programme - in einem opportunistischen Rahmen durchgeführt werden. Dass die Qualität unter diesen Umständen weniger gut kontrolliert werden kann, ist hinlänglich bekannt. Und wozu alle Formen des Mammographie-Screenings bezüglich Qualität evaluiert werden sollen, wenn die Nachteile so klar überwiegen sollen, ist uns nicht klar.

\section{Where have all the experts gone?}

Häufig folgt der Veröffentlichung eines SMB-Berichts ein Klagelied der Unzufriedenheit. Dies erstaunt insofern, als doch die SMB-Regelungen zu Organisation und Prozessen [5] vorsehen, externe Fachspezialisten beizuziehen, damit diese ihr spezifisches Fachwissen einbringen können. Dies wurde auch getan und die Experten im Bericht erwähnt. Umso mehr erstaunt es, dass einige der befragten Experten ihre Aussagen und Argumente in keinerlei Weise im Bericht vorfinden [6]. Insbesondere hat das SMB die Anregung der Experten, auch die Evaluationen von Programmen zu berücksichtigen, nicht aufgenommen. Dies wäre insbesondere bei Interventionen auf Public Health-Ebene mit langfristiger Perspektive - und dazu gehört das Mammographie-Screening - eine sinnvolle Ergänzung zu den (in diesem Bereich alten) randomisierten Studien. hat) sind aufgefordert, ihre Haltung nochmals im Rahmen der Post-Publication Review einzubringen. Ob dies die Mühe wert ist? Offenbar nicht.

Die Veröffentlichung der Post-Publication Review am 12. August 2014 [7] bestätigt die Zweifel: Die Review stellt eine nahtlose Weiterführung des ursprünglichen Berichts dar. Betrachtet man die Zusammenfassung der Review, könnte man den Eindruck gewinnen, dass mehr Zustimmung als Kritik zum Bericht des SMB besteht. In der Zusammenfassung reduziert sich die in der Debatte geäusserte Kritik auf den Satz «Divergierende Meinungen gibt es insbesondere zur Interpretation der Datenlage und welche Schlussfolgerungen aus dieser Interpretation gezogen werden sollen». Die Kritik am methodischen Vorgehen unter «Interpretation der Datenlage» zu subsummieren, scheint uns doch ein bisschen zu einfach. Beispielsweise ist der KarnofskyIndex zur Berechnung der QALYs inadäquat und unüblich. Daran wird auch das unbeirrte Festhalten des SMB nichts ändern.

Es erstaunt nach dieser Vorgeschichte nicht, dass das Fachgremium SMB aufgrund der «unveränderten Sachlage» an den Empfehlungen festhält. Die von einigen Exponenten des SMB in Aussicht gestellte Überarbeitung des Berichts dürfte damit wohl Geschichte sein.

\section{Wir sind der Ansicht, dass das SMB die positiven Auswirkungen des Mammographie-Screenings unterschätzt und die unerwünschten Auswirkungen überschätzt.}

Bemerkenswert ist auch, dass sich der ebenfalls befragte Verband «swiss cancer screening» nach Feststellung der fehlenden Berücksichtigung aus der Liste der zur Erarbeitung beigezogenen Experten streichen liess und nun als «kontaktierte Organisation» in einer Fussnote erwähnt wird. Von einer professionellen Verfasserin des Berichts wäre zu erwarten, dass die Expertenmeinungen transparent aus dem Bericht hervorgehen und nicht nach Gutdünken ignoriert werden.

Ein echter Einbezug der Experten hätte auch Fehler wie die laienhafte Vermischung der falschpositiven Befunde und der Überdiagnosen unter dem Begriff «Fehlbefunde» verhindert. In dubio pro reo, dass dies nicht mit Absicht erfolgt ist.

\section{Abstellgleis Post-Publication Review?}

Seit kurzer Zeit ruft das SMB zu sogenannten PostPublication Reviews auf. Organisationen und Fachexperten werden dazu eingeladen, ihre Haltung zum Bericht nachträglich einzureichen.

Die befragten Experten (deren Meinung wie oben erwähnt keinen Eingang in den Bericht gefunden

\section{Fazit: Eine Revision des SMB ist notwendig}

Das SMB analysierte einseitig das MammographieScreening in Programmen, ohne neue wissenschaftliche Daten zu berücksichtigen, ohne Einbezug der Meinung befragter Experten, ohne Einbezug der Realität in der Schweiz mit der hohen Anzahl opportunistischer Untersuchungen, stattdessen mit einer nicht nachvollziehbaren Kosten-Nutzen-Analyse und widersprüchlichen Empfehlungen. Sind diese Unzulänglichkeiten auf die Zusammensetzung des SMB zurückzuführen, das nicht über das notwendige methodologische und klinische Wissen zu verfügen scheint?

Auch wir sind der Meinung, dass das Mammographie-Screening kontinuierlich weiterentwickelt werden soll und medizinisches Handeln wenn immer möglich evidenzbasiert erfolgen soll. Dazu braucht es jedoch qualitativ gute und differenzierte Grundlagen. Neben den randomisierten Studien, die in diesem Fall jedoch nicht die heutige Realität abbilden, müssen dazu auch aktuelle Erkenntnisse aus den Mammographie-Screening-Programmen berücksichtigt werden. Der Bericht des SMB zum Mammographie-Screening erfüllt diese Anforderungen nicht. 


\section{Beurteilung des SMB-Berichts zum Mammographie-Screening durch Harry de Koning*}

Die Kosten-Wirksamkeits-Analyse des SMB-Berichts zum Mammographie-Screening weist in verschiedenen Aspekten gravierende Mängel auf: Insgesamt werden die positiven Auswirkungen des Screenings unterschätzt, die negativen Auswirkungen überschätzt. Die wichtigsten Mängel des Berichts sind die folgenden:

- Bei der Abschätzung der Anzahl verhinderter Todesfälle stützt sich das SMB nicht auf die aktuellen Zahlen zu Brustkrebstodesfällen in der Schweiz, sondern auf die Daten aus den randomisierten Studien der 1980er-Jahren. Methodisch adäquat wäre gewesen, analog zur englischen Expertengruppe in ihrem Review [3] aktuelle absolute Zahlen zu verwenden. Durch dieses Vorgehen des SMB werden die positiven Auswirkungen im Sinne der verhinderten Todesfälle unterschätzt.

- Die Berechnungen des SMB decken einen Zeitraum von nur 13 Jahren ab. Methodisch adäquat wäre gewesen, die Auswirkungen des Screenings über die gesamte Lebensspanne der Frauen zu berücksichtigen. Die positiven Auswirkungen des Screenings im Sinne von gewonnenen Lebensjahren durch verhinderte Todesfälle werden dadurch stark unterschätzt.

Ähnliche Rückmeldungen erfolgten auch zu anderen Berichten des SMB (vergleiche z. B. SÄZAusgabe 29-30/2014 [8]). «Das Swiss Medical Board genügt nicht» so titelte Michael Schlander seinen Gastkommentar vom 14. November 2013 in der NZZ [9]. Auch wir unterstützen darum die Bestrebungen des Trägervereins, die «Strukturen wie auch das Verfahren zu verbessern» [10], in der Hoffnung, dass dies zu qualitativ guten HTA-Analysen in der Schweiz beitragen wird.

\section{Interaktiver $::: .:$ Literatur \\ Artikel}

Wollen Sie diesen Artikel kommentieren? Nutzen Sie dafür die Kommentarfunktion in der OnlineVersion oder sehen Sie nach, was Ihre Kolleginnen und Kollegen bereits geschrieben haben: www.saez.ch/ aktuelle-ausgabe/ interaktive-beitraege/
- Während Screening-Programme einen Zeitraum von 20 Jahren abdecken (was bei einem Intervall von zwei Jahren zehn Mammographien pro Frau entspricht), geht das SMB in seinen Berechnungen von drei Mammographien pro Frau aus. Damit werden die positiven Auswirkungen des Screenings im Sinne von gewonnenen Lebensjahren unterschätzt.

- Die Kosten-Wirksamkeits-Analyse berücksichtigt nicht alle relevanten Auswirkungen durch Screening-Programme, insbesondere den Rückgang von Diagnosen in fortgeschrittenem Stadium.

- Durch die Limitierung auf eine Beobachtungszeit von 13 Jahren werden die negativen Auswirkungen des Screenings durch Überdiagnosen überschätzt.

- Die negativen Auswirkungen durch falsch-positive Befunde werden durch die Annahme einer starken Beeinträchtigung der Lebensqualität über mehrere Monate überschätzt.

Harry de Koning, Professor of Evaluation of Screening, Erasmus MC, Rotterdam

* Zusammenfassung des Artikels: 21st century science on the back of a coaster - A review of the Swiss Medical Board's «Systematisches Mammographie-Screening» report (2014), by $\mathrm{H}$. J. de Koning, MD PhD (submitted for publication).

5 Swiss Medical Board. Organisation und Prozesse des Medical Board. Stand vom 31. Januar 2011, abgerufen am 1. Juli 2014 unter www.medical-board.ch/ fileadmin/docs/public/mb/methodik/2011-01-31_ Organisation_und_Prozesse.pdf

6 Swiss Society of Senology. SMB Mammography Screening Recommendations: Concerns shared Conclusions wrong. Schweiz Ärztezeitung. 2014;95(36):1315-6.

7 Swiss Medical Board. Systematisches MammographieScreening - Dokumentation und Würdigung der Stellungnahmen zum Fachbericht (Post-PublicationReview). Veröffentlicht am 12. August 2014.

8 Arbeitsgruppe Lipide und Atherosklerose (AGLA), der Schweizerischen Gesellschaft für Kardiologie (SGK), und der Schweizerischen Gesellschaft für Herz- und Gefässchirurgie (SGHC). Statine zur Primärprävention kardiovaskulärer Erkrankungen. Schweiz Ärztezeitung. 2014;95(29/30):1084-6.

9 www.nzz.ch/meinung/debatte/warum-das-swissmedical-board-nicht-genuegt-1.18185108

10 Swiss Medical Board. Jahresbericht 2013. 